\title{
Trends of Severe Acute Malnutrition Morbidity and Mortality (2014-2017), Bale Zone, Oromia Region, Ethiopia, 2018
}

\author{
Mohammed Hasen (D) \\ Henok Asefa ${ }^{2}$ \\ Naod Berhanu ${ }^{3}$ \\ Falaho Sani $\left.{ }^{1}\right)^{\prime}$ \\ 'Field Epidemiology and Laboratory \\ Training Program, Faculty of Public \\ Health, Jimma University, Jimma, Ethiopia; \\ ${ }^{2}$ Departments of Epidemiology, Faculty of \\ Public Health, Jimma University, Jimma, \\ Ethiopia; ${ }^{3}$ Field Epidemiology and \\ Laboratory Training Program, Jimma \\ University, Jimma, Ethiopia
}

Correspondence: Mohammed Hasen

P. O. Box: 6I, Gindhir, Ethiopia

Tel +251923728803

Email direhasen@gmail.com
Background: Malnutrition is a serious disease and remains an important public health problem in many developing countries including Ethiopia. Malnutrition is one of the diseases under the surveillance system, which is reported weekly and monthly. In the Bale zone, malnutrition is one of the major public health problems. Therefore, this trend analysis of severe acute malnutrition (SAM) was used to describe the magnitude, trends, disease outcome and geographical distribution of the SAM in Bale Zone from 2014 to 2017.

Methods: A descriptive cross-sectional study was conducted in April 2018. Data were extracted from the Bale zone's monthly malnutrition report database and checked for completeness and consistency; then, four years' worth of trends of SAM were analyzed. The prevalence and trend of SAM by Woreda, year and age were analyzed using Microsoft Office Excel 2016 and SPSS version 20 then summarized using text, tables, and figures.

Results: A total of 37,678 SAM cases were registered over four years (2014-2017). Of these, 31,642 (84\%) were cured, $52(0.14 \%)$ died, $641(1.7 \%)$ were defaulters, and 66 $(0.18 \%)$ were non-respondents. Among registered cases, $98.2 \%$ are in the 6-59 months age groups. The average annual prevalence of SAM high among under-five children was $3.3 \%$. The prevalence rates per 1000 people suffering from SAM in 2014, 2015, 2016, and 2017 are 3.6, 4.5, 7.5, and 4.7 respectively, and the death rates were $0.21 \%, 0.26 \%, 0.1 \%$, and $0.08 \%$, respectively. Among the Woreda, the highest prevalence was at Delomena $(2.45 \%)$ and the lowest was at Sinana woreda $(0.1 \%)$. The death rate is high among the 659 months age groups $(0.13 \%)$ and followed by the 6 months of group $(0.008 \%)$.

Conclusion: Cases of SAM increased between 2014 and 2016 and then decreased during 2017. The prevalence of SAM was high in the under-five age group of children in the Bale zone during the study period and the Delomena Woreda has the highest average annual prevalence of SAM. We recommend further research on why some districts have a high prevalence of SAM.

Keywords: malnutrition, trends, Bale zone

\section{Introduction}

Malnutrition is a general term for a medical condition caused by an inappropriate or inadequate diet. The term usually refers to generally faulty nutrition and most often related to undernutrition. ${ }^{1}$ The number of children aged under five who are chronically or acutely undernourished (wasted) may have fallen in many countries. However, the data tracking shows that global progress to scale back these varieties of nutrition deficiency disease is not fast enough to fulfill internationally united nutrition targets, including Sustainable Development Goal (SDG), target 2.2, to end 
all forms of malnutrition by 2030 . Hunger statistics might be sometimes be heading in the wrong direction: currently 815 million people are going to bed hungry, up from 777 million in 2015. The reality of famines within the world these days means that achieving these targets, especially for wasting, will become even more challenging. Indeed, an estimated 38 million people are facing severe food insecurity in Nigeria, Somalia, South Sudan, and Yemen, while Ethiopia and Kenya are experiencing significant droughts. ${ }^{2}$

According to the global burden of diseases study, malnutrition attributes to $11.7 \%$ of deaths and $15.9 \%$ of DALY globally, and are the most significant contributors to the risk factors of the global burden of the diseases. ${ }^{3}$

According to the 2016 Ethiopia Demographic Health Survey (EDHS), 38\% of children under 5 age are stunted or too short for their age, and $18 \%$ are severely stunted. Ten percent are wasted or too thin for their height, including 3\% who are severely wasted. Twenty-four percent of children under the age of 5 are underweight or are too thin for their age, with $7 \%$ being severely underweight. The prevalence of overweight children remained low at $1 \%{ }^{4}$

Different problems are responsible for the child being undernourished, especially in developing countries. Of these problems, some basic issues are political instability, slow economic growth, and lack of education. Others are underlying causes such as food insecurity and lack of maternal and childcare services. The third group includes the highly specific risk factors, such as frequent infections and inadequate dietary intake. ${ }^{5}$

Conducting trend analysis is a key operation for monitoring illness trends, and evaluating the effectiveness of disease management programs and policies. Results from trends of severe acute malnutrition (SAM) can trigger public health action when the prevalence of diseases is increasing and to describe the epidemiology of SAM. Even though malnutrition is one of the major public health problems in the Bale zone, trends of SAM have not been analyzed. Therefore, this study aimed to assess trends of SAM morbidity and mortality from 2014-2017 in Bale zone, Oromia Region.

\section{Materials and Methods Study Area}

Bale is one of the zones in the Oromia region, Ethiopia, which administratively divided into 18 Woreda and two towns. Bale zone is $430 \mathrm{~km}$ from Addis Ababa located at in a south-east direction, with an estimated total population of 1,858,639 in 2017 and a total area of $67,329.59 \mathrm{~km}^{2}, 14 \%$ of which is highland and $64 \%$ is lowland. Among the total Woreda, nine are pastoralist and the remaining nine were agro-pastoralists. The zone shares boundaries with the Somali region, West Arsi, Guji zone, West Hararge, Arsi on the east, west, south, north-west, and North, respectively. In 2017 there were four hospitals, 82 health centers and 381 health posts in the Bale Zone. All health facilities provide SAM treatment services.

\section{Study Design and Period}

A cross-sectional study was conducted. We reviewed four years (2014-2017) of SAM secondary data at the Bale zone health office in April 2018. All SAM data was available from the Bale zone, Nutrition department.

\section{Case Definition}

\section{Severe Acute Malnutrition}

For infants aged less than 6 months or weighing less than $3 \mathrm{~kg}$ : Weight-for-Length (WFL) was less than $70 \%$ or $<-3 Z$ score or had the prescence of pitting edema on both feet or visible severe wasting where it was difficult to determine WFL.

For children aged 6 months to 18 years: Weight-forLength (WFL)/WFH less than $70 \%$ or $<-3 \mathrm{Z}$ score or presence of pitting edema on both feet or MUAC $<11 \mathrm{~cm}$ for child length greater than $65 \mathrm{~cm}$.

For adults: MUAC was $<180 \mathrm{~mm}$ with a recent loss or underlying chronic illness or MUAC $<170 \mathrm{~mm}$ or BMI $<16$.

\section{Source of Data and Collection Procedure} Secondary data of SAM from routine Public Health Emergency Management (PHEM) surveillance system and nutrition department were reviewed from Bale zonal databases. Data was computerized at the Bale zone health office in PHEM surveillance system and malnutrition department. In the study area, severely acute malnutrition patients were treated at the health post, health center and hospital according to the Ethiopian malnutrition national guideline. ${ }^{6}$ Data for variables like a month, year, Woreda, age, treatment outcomes was extracted from the reporting format. Accordingly, four years' SAM secondary data (2014-2017) was extracted from Bale zonal health office malnutrition report database using a prepared data extraction format.

\section{Data Analysis Procedure}

All data were checked for completeness and consistency. Data were analyzed by place, person and time using Microsoft Office Excel 2016 and SPSS version 2020. 
The retrospective data of SAM was summarized using figures, tables, line graphs and bar charts.

\section{Ethics}

We obtained ethical approval from Jimma's ethical review board and an official letter from Bale zone Health Department (reference number BFG/14463) to use secondary data of malnutrition.

\section{Results}

\section{Severe Acute Malnutrition by Time}

A total of 37,678 SAM cases were registered over a 4 year period (2014-2017). Of these, 33,540 (89\%) of SAM cases were treated at the Outpatient Therapeutic Program (OTP) and $4138(11 \%)$ of the cases were treated at stabilization center (SC) programs. The prevalence of SAM in 2014, 2015, 2016, and 2017 was 3.6, 4.5, 7.5, and 4.7 per 1000 people, respectively (Figure 1), and death rates were $0.21 \%$, $0.26 \%, 0.1 \%$, and $0.08 \%$, respectively. The prevalence of SAM, on average, increased $0.11 \%$ per year in the Bale zone. Cases of SAM admitted to OTP increased by $26.1 \%$ from 2014 to 2015 and increased by $70 \%$ in 201-2016 and decreased by $36.2 \%$ in $2016-2017$ and similarly, cases of malnutrition admitted to SC increased by $55.6 \%$ from 2014 2015 and increased by $71.3 \%$ from 2015-2016 and decreased by $27.2 \%$ in 2016-2017 (Figure 2).

\section{Severe Acute Malnutrition by Place}

From the total registered cases of SAM, the majority of the cases $(4646 ; 12.3 \%)$ were reported from Delomena
Woreda, followed by Berbere woreda (3636; 9.7\%) and Goro woreda $(3389 ; 8.99 \%)$. The lowest cases of SAM during this period were reported from Goba rural which had $228(0.6 \%)$ cases (Figure 3).

\section{Severe Acute Malnutrition by Person}

From the total registered cases of SAM, 37,027 (98.2\%) cases were 6- to 59-month-old children, followed by 293 $(0.8 \%)$ cases in children under 6 months of age and 194 $(0.5 \%)$ cases were in $5-10$ years' children (Table 1$)$. The prevalence of SAM in the 6-59 months age group in 2014, $2015,206,2017$, are $2.61 \%, 3.06 \%, 4.66 \%$, and $3.10 \%$ respectively and the average annual prevalence was $3.3 \%$.

Among the total admission of SAM cases, 23,530 $(62.5 \%)$ were cured and $48(0.13 \%)$ deaths from the 659 months group, followed by infants less than 6 months of age, of whom, $236(0.63 \%)$ were cured and $3(0.008 \%)$ died. The death rate in 2014, 2015, 2016, and 2017 were $0.21 \%, 0.26 \%, 0.1 \%$, and $0.08 \%$ respectively.

\section{Discussion}

SAM is one of the most common causes of morbidity and mortality worldwide. ${ }^{6}$ It is also a public health problem in Ethiopia, especially in an area affected by drought, like the Bale zone in the Oromia region. ${ }^{7}$ This study describes the trends of SAM morbidity and mortality in the Bale zone between 2014-2017.

The prevalence of SAM in the Bale zone increased continuously from 2014 to 2016 . This study finding is consistent with the finding reported by the humanitarian

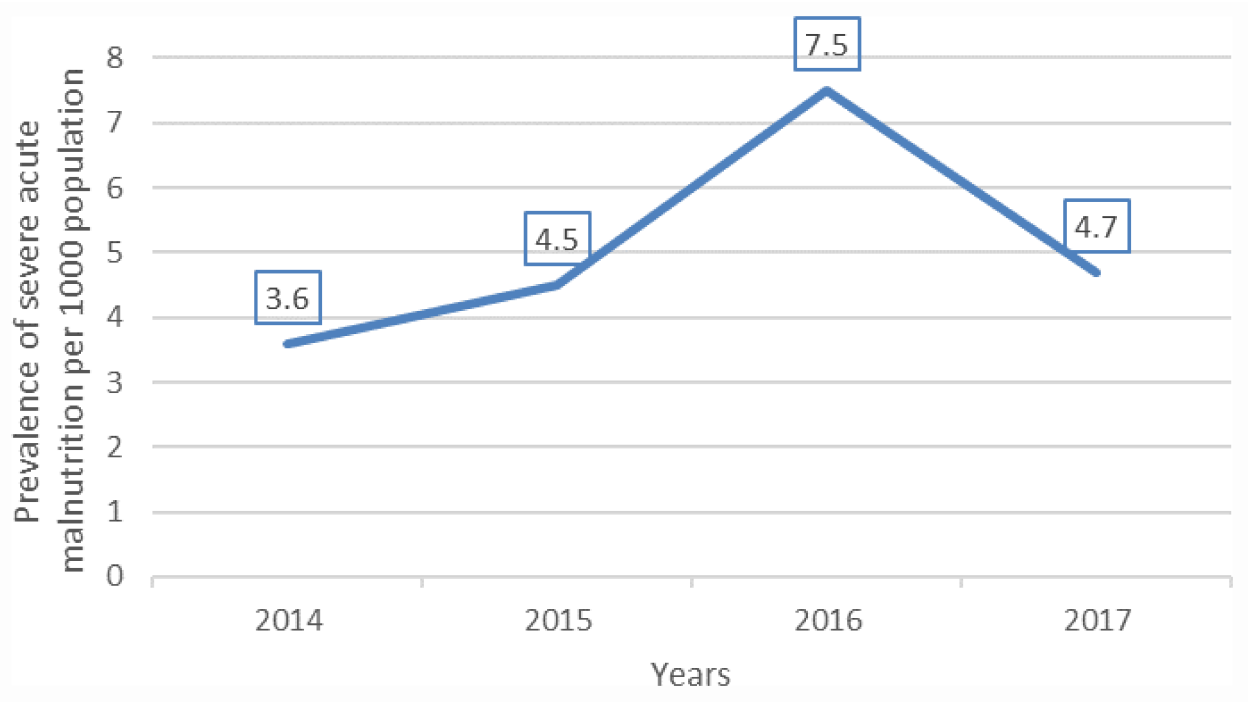

Figure I Prevalence of SAM from 2014-2017 in the Bale zone, Oromia, Ethiopia, April 2018. 


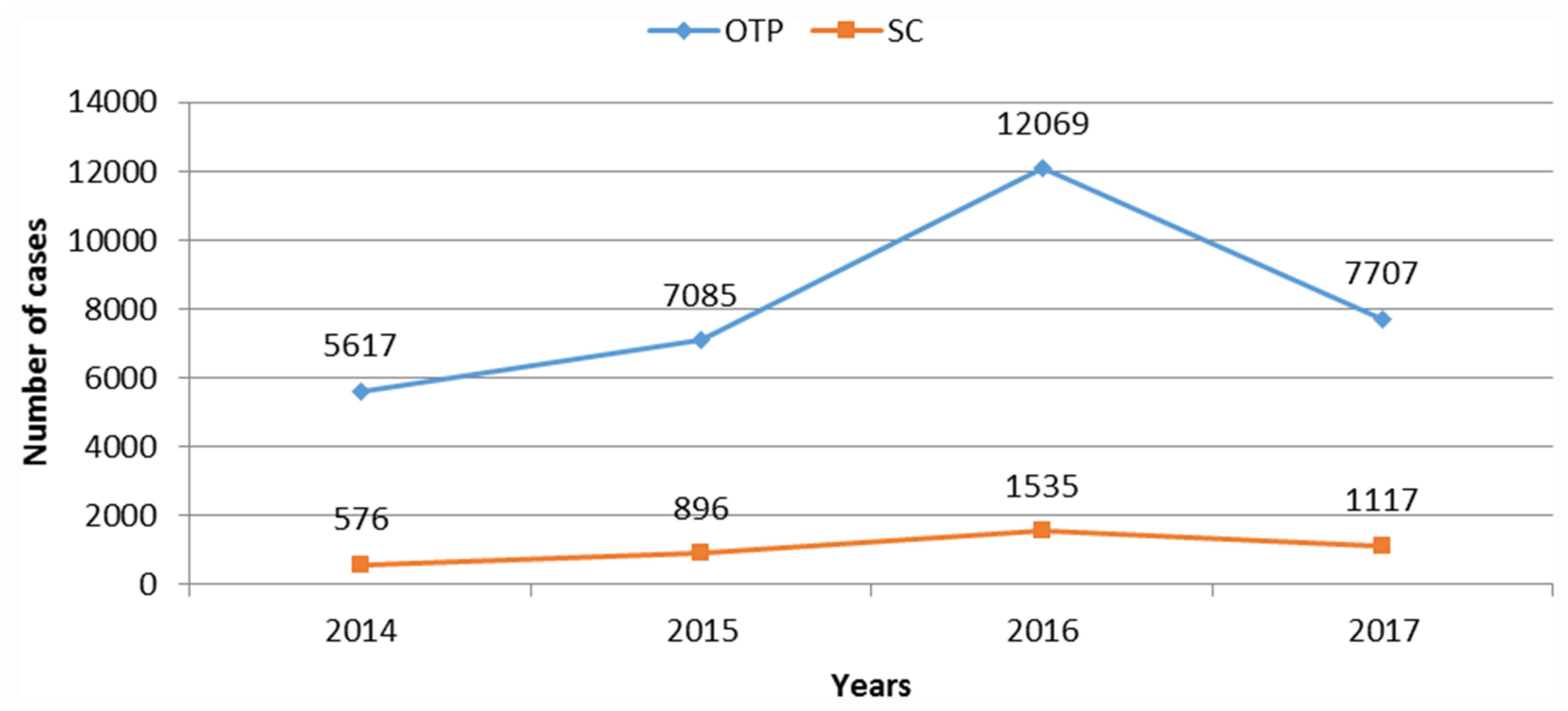

Figure 2 Total new admissions of SAM from 2014-2017 in the Bale zone, Oromia, Ethiopia, April 2018.



Woreda

Figure 3 SAM by woreda from 2014-2017 in the Bale zone, Oromia, Ethiopia, April 2018.

situation report. This may be associated with an increased number of outpatient therapeutic feeding program sites and screening services conducted at the community level. The death rate increased by the year to 2015 , but substantially decreased between 2015 and 2017. These were due to the intervention program conducted with the expansion of OTP and SC sites. ${ }^{7}$
The prevalence of SAM in under-five year children markedly higher than that of above -years-old. This finding is consistent with the scientific background which indicated that children under 5 are more affected by malnutrition. ${ }^{6,8}$ Additionally, our finding might be due to the high participation rate of children under5 on malnutrition screening because of government policy highly focus 
Table I Total Registered SAM Cases by Age from 2014 to 2017 at Bale Zone, Oromia Region, Ethiopia, April 2018

\begin{tabular}{|l|l|l|l|}
\hline Cases by Age & OTP* & SC** & Total (\%) \\
\hline$<6$ months & 9 & 284 & $293(0.8)$ \\
$6-59$ months & 33,315 & 3712 & $37,027(98.2)$ \\
$5-10$ years & 63 & 131 & $194(0.5)$ \\
$11-17$ years & 97 & 10 & $107(0.3)$ \\
$>18$ years & 54 & 3 & $57(0.15)$ \\
\hline
\end{tabular}

Notes: *Cases admitted to outpatients; **cases admitted to stabilization centers.

on these age groups. ${ }^{9}$ Therefore, this finding implies that children under 5 suffered from SAM. The average annual prevalence of SAM of children under 5 from 2014-2017 are almost equal with that of the Oromia region according to EDHS-2016. ${ }^{4}$ The prevalence of SAM in children under 5 (3.1\%) in 2017 was less than that of study the conducted at the Shashemene referral hospital $(25.2 \%),{ }^{10}$ and a study conducted at east Hararge Haramaya district $(14.43 \%){ }^{11}$ These are likely due to study population, our study was conducted at the zonal level and the population of the Bale zone was our study group, unlike the study conducted at Shashamane hospital which is facility-based.

The prevalence of malnutrition higher in the rural area when compared to town at the Bale zone, this is comparable with the EDHS 2016. ${ }^{4}$ Dello mena woreda has the highest average annual prevalence of SAM, followed by Meda Welabu and Harena buluk. However, Gasera and Sinana woreda has the lowest average annual prevalence of SAM.

This study has its limitations. First it was limited by the being a retrospective study, as we were relying only on already collected secondary data. There was a possibility that some of the records may not be correct. Second, we were limited to the variable on the reporting format. However, our study revealed important findings, such as Woreda with the high prevalence of SAM have been identified, so these are the priority areas for the further research.

\section{Conclusion}

The prevalence of SAM increased by $0.11 \%$ annually in the Bale zone. The prevalence was high among children under 5. The prevalence of SAM varied among Woredas of the zone and high at Delomena Woreda. The case fatality rate and defaulters decreased during the 4 years of the study period. The Bale zonal health office and other stakeholders should strengthen their nutrition programs to tackle the increment trends of increasing SAM Additionally, we recommend further research to investigate the reasons for the high prevalence of SAM in some Woreda of Bale zone.

\section{Abbreviations}

EDHS, Ethiopia Demographic Health Survey; MUAC, Mid Upper Arm Circumference; OTP, Outpatient Therapeutic Program; SC, Stabilization Center; SPSS, Statistical Package for the Social Sciences; WFL, Weight -for- Length.

\section{Data Sharing Statement}

All the data supporting our findings of this study are available from the corresponding author upon request.

\section{Ethical}

The study used existing secondary data of severe acute malnutrition extracted from Bale Zone Health Office nutrition report database. We obtained the ethical approval from Jimma ethical review board and Permission to use data and conduct the study has obtained from Bale zone health department by the reference number BFG/14463. Confidentiality of the data was assured and was used to this study only. The data accessed complied with relevant data protection and privacy regulations.

\section{Acknowledgments}

We would like to acknowledge Jimma University for their support letter and Ethiopia field epidemiology and laboratory training program. In addition, we would like to thanks the Bale Zone health office, public health emergency management department and malnutrition department for their given permission to us to analyze data.

\section{Disclosure}

The authors declare that they have no conflicts of interest in this work.

\section{References}

1. Nikolaos K, Charilaos D, Meropi K, Evangelia M. Clinical Nutrition in Practice. Athens, Greece: A John Wiley \& Sons, Ltd, Publication; 2010.

2. Hawkes C, Fanzo J, Udomkesmalee E. Global nutrition report 2017: nourishing the SDGs. Bristol, UK; 2017.

3. Christopher JL, Lopez AD. Global burden of disease and injur y series the global burden of disease. A comprehensive assessment of mortality and disability from diseases, injuries, and risk factors in 1990 and projected to 2020. Boston, MA, USA; 1996. 
4. Central Statistical Agency (CSA) [Ethiopia] and ICF. Ethiopia Demographic and Health Survey 2016; 2016.

5. Gudina E, Yemane B, Alemayehu W. Seasonal variation in the prevalence of acute undernutrition among children under five years of age in east rural Ethiopia: a longitudinal study. BMC Public Health. 2013;1:1.

6. Ethiopia Federal Ministry of Health. Training course on the management of severe acute malnutrition (guidline) Federal democratic Republic of Ethiopia. Addis Ababa, Ethiopia: Ministry of Health; 2013.

7. UNICEF. Ethiopia Humanitarian Situation Report 4, UNICEF Ethiopia. Addis Ababa, Ethiopia; 2016.
8. Md Behrman RE, Md Kliegman RM, Md Jenson HB, Editors. Nelson Textbook of Pediatrics 17th Edition (May 2003). W B Saunders; May, 2003. OkDoKeY.

9. Ethiopia Ministry of Health. Health Sector Transformation Plan. Federal Democratic Republic of Ethiopia Ministry of Health, 2015/16-2019/20. Addis Ababa, Ethiopia; 2015.

10. Kassa ZY, Behailu T, Mekonnen A, Teshome M. Malnutrition and associated factors among under five children (6-59 Months). Curr Pediatr Res. 2017;21:172-180.

11. Fuad F, Gudina E, Adem K. Prevalence of malnutrition among children aged 6-59 in Haramaya. J Biom Biostat. 2017;4:8-11.

\section{Publish your work in this journal}

Nutrition and Dietary Supplements is an international, peerreviewed, open access journal focusing on research into nutritional requirements in health and disease, impact on metabolism and the identification and optimal use of dietary strategies and supplements necessary for normal growth and development. The journal welcomes submitted papers covering original research, basic science, clinical \& epidemiological studies, reviews and evaluations, guidelines, expert opinion and commentary, case reports and extended reports. The manuscript management system is completely online and includes a very quick and fair peer-review system, which is all easy to use. Visit http://www.dovepress.com/testimonials.php to read real quotes from published authors. 